\title{
ÉTUDE TECHNIQUE SUR LA FONTE DU MIEL CRISTALLISÉ
}

\author{
PAR
}

\section{J. LOUVEAUX et E. TRUBERT}

Station de Recherches Apicoles, Bure-sur-Yvette

I1 est rare que la totalité de la récolte de miel d'un apiculteur professionnel soit répartie en emballages de détail aussitôt après extraction et maturation. Bien souvent intervient une phase de stockage en récipients d'importance variable allant du seau de $20 \mathrm{~kg}$ au fût de $300 \mathrm{~kg}$ dans lesquels se produit presque toujours la cristallisation spontanée du miel.

Pour pouvoir répartir en emballages de détail le produit cristallisé, il est indispensable de le réchauffer suffisamment pour le ramener sinon à l'état parfaitement liquide, du moins à l'état de suspension de cristaux dans une masse liquéfiée. C'est dans cet état, obtenu facilement vers $45^{\circ}$, que le miel se prête le mieux à une mise en pots facile et à une recristallisation rapide et relativement homogène.

I a technique de refonte du miel cristallisé est susceptible à notre avis de perfectionnements multiples portant à la fois sur la conservation des propriétés biologiques et diététiques du miel, facilement altérables par une élévation de température exagérée, et aussi sur le prix de revient de l'opération. En dehors de ces considérations il convient également de tenir compte des facilités de manipulation que l'on peut apporter par une organisation rationnelle du travail.

Étude théorique du problème. - Nous rappellerons tout d'abord quelques données élémentaires relatives à l'aspect théorique du problème de la fonte du miel cristallisé. I,iquide au moment de la récolte, le miel ne tarde pas à la température ordinaire (1 $8^{\circ}$ ) à se transformer en une masse solide plus ou moins consistante qui est un feutrage de cristaux constitués surtout de glucose mais probablement aussi d'une faible proportion de lévulose ou de complexes glucose-lévulose. Ce feutrage retient entre ses mailles une phase liquide qui est constituée surtout par un sirop de lévulose. Toute élévation de température provoque le retour en solution de la partie cristallisée. Ce changement d'état est progressif et l'on peut trouver tous les intermédiaires entre le miel parfaitement solide tel 
qu'il se présente à I $8^{\circ}$ et le miel entièrement liquéfié, limpide et dépourv'u de germes de cristallisation tel qu'on l'obtient entre $55^{\circ}$ et $60^{\circ}$.

Si nous suivons sur un petit échantillon de $25^{\circ} \mathrm{g}$ de miel placé dans une étuve réglée à $60^{\circ}$ le processus de liquéfaction, nous pouvons noter un certain nombre d'étapes (fig. I). Un thermonètre placé an centre de l'échantillon nous donne la température qui règne clans le miel. A $23^{\circ}$ le miel est ferme ; à $27^{\circ}$ il est pâteux et commence à couler lorsqu'on incline le récipient. A $30^{\circ}$ il est pâtenx très conlant. A $3 t^{\circ}$ on observe

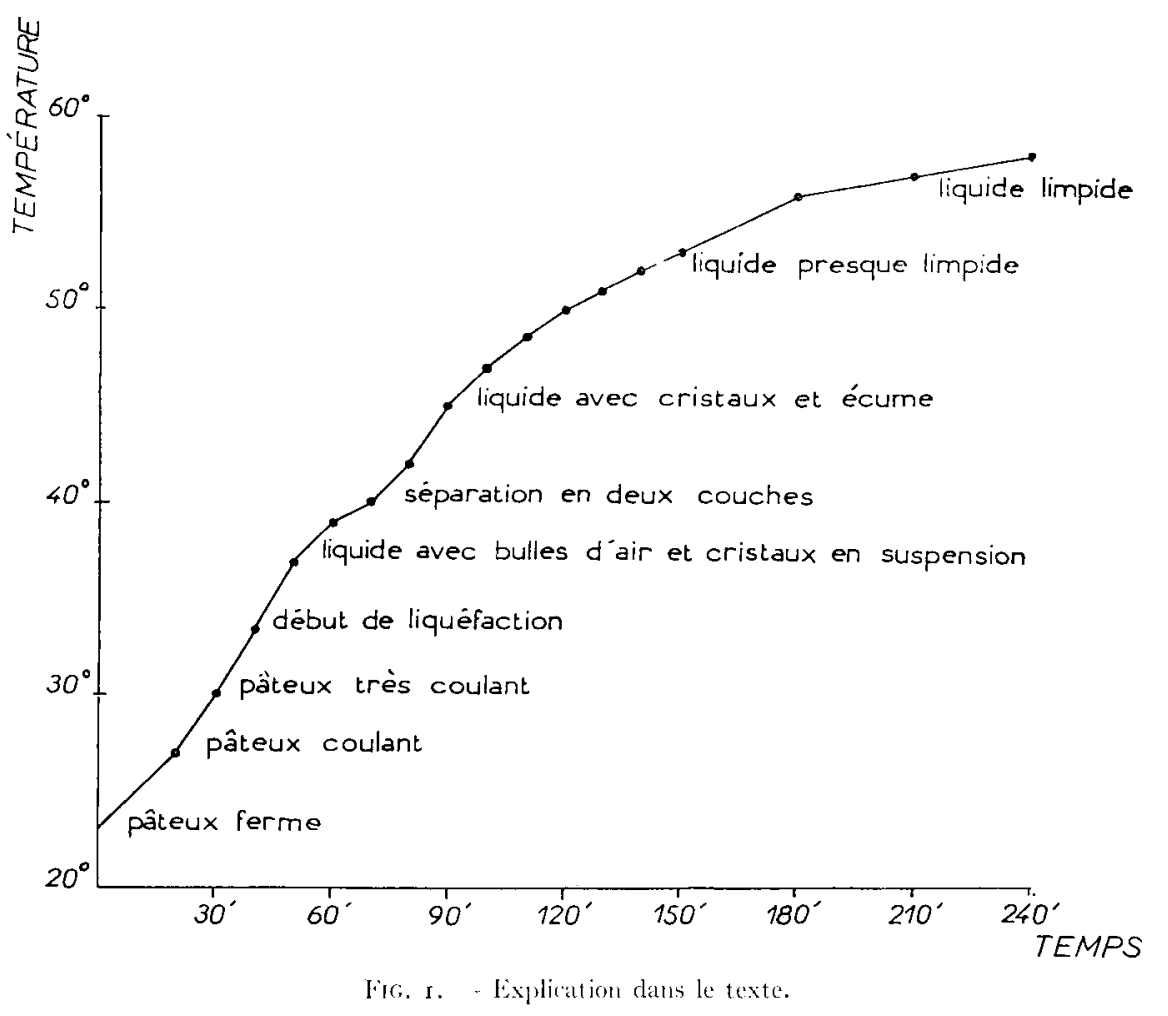

un début de liquéfaction à la partie inférieure tandis qu'une remontée d'écume commence à se produire. A $37^{\circ}$ le miel est liquide et coule parfaitement mais il est chargé de bulles d'air et d'une infinité de cristaux non encore dissous. Jusqu'à $50^{\circ}$ on observe la remontée progressive des bulles d'air qui finissent par faire en surface une couche d'écume tandis que les cristaux disparaissent. Vers $55^{\circ}$ 1a clarification du miel est parfaite et aucune élévation de température supplémentaire ne modifie son état physique.

Un miel réchauffé uniformément à $55^{-60^{\circ}}$ ne recristallise que très lentement, surtout s'il reste à l'abri de l'air en récipients bien clos. Selon sa composition chimique il reste indéfiniment liquide ou bien il manifeste 
au bout de quelques mois au plus une recristallisation très lente à gros cristaux irréguliers du plus fâcheux effet. Un miel réchauffé uniformément à $43-45^{\circ}$ devient suffisamment liquide pour pouvoir être transvasé mais il conserve suffisamment de cristaux en suspension pour qu'une recristallisation correcte intervienne dans les quelques semaines qui suivent l'opération, surtout si la conservation se fait aux environs de $\mathrm{I}_{4}^{\circ}$.

I'opération idéale de refonte du miel consisterait donc en un réchauffage progressif jusqu'à $45^{\circ}$ sans qu'aucun point de la masse du miel ne soit jamais chauffé au-delà de cette température. Ceci suppose une étuve réglée à $45^{\circ}$ et un temps de réchauffement relativement long. Fin effet, une masse de miel froide placée dans une enceinte chauffée tend à se mettre en équilibre thermique avec cette enceinte qui lui cède des calories. La vitesse des échanges est proportionnelle à l'écart des températures, c'est-à-dire que l'échauffement est d'autant plus lent qu'on se rapproche davantage de la température finale qui est celle de l'enceinte. Un réchauffement dans une enceinte maintenue à la température finale désirée sera donc forcément long. Si l'on veut opérer vite il faut porter l'enceinte à une température nettement supérieure à la température finale désirée et arrêter le réchauffement au monent où le miel a emmagasiné assez de calories pour pouvoir se trouver, après répartition des calories à l'intérieur de la masse, à la température finale choisie. Au cours du réchauffage rapide une partie du miel peut se trouver portée à une température très supérieure à celle que l'on désire finalement obtenir. C'est par échanges internes de calories qu'on aboutit finalement à une température convenable.

Une opération de refonte d'un poids donné de miel devrait pouvoir se calculer entièrement et sans qu'il soit nécessaire de procéder à une étude empirique préalable. Mais un tel calcul, s'il est possible théoriquement, suppose la connaissance parfaite de certaines données importantes qui n'ont pas fait jusqu'ici l'objet d'études suffisantes. Le premier facteur à intervenir est la conductibilité thermique du miel. Elle a été calculée par Hel,vey (I) pour un miel finement granulé ; cet auteur a trouvé à $20^{\circ}$ :

$$
\lambda=0,00129 \mathrm{cal} / \mathrm{cm} \mathrm{sec}{ }^{\circ} \mathrm{C} \text {. }
$$

ce qui correspond à peu près à la conductibilité calorifique d'un bon isolant tel que le verre mais non d'un calorifuge tel que le liège $(\lambda=0,000 \mathrm{I})$. I, a cire d'abeilles a un $i$ un peu plus faible $(0,00092)$ que celui du miel. I a conductibilité thermique varie avec la teneur en eau du miel et avec la température. On est assez mal renseigné sur la conductibilité thermique du miel liquide ainsi que sur celle du miel imparfaitement cristallisé contenant une phase solide et une phase liquide importante. Il apparaît donc assez difficile de connaître la vitesse exacte d'échauffe- 
ment d'un miel ; ceci est d'autant plus vrai qu'au fur et à mesure du réchauffement la proportion de miel solide change constamment. Des courants de convexion apparaissent dans le miel liquéfié, ce qui ne contribue pas à simplifier le problème. Ainsi que nous l'avons vérifié expérimentalement une masse de miel solide que l'on réchauffe se liquéfie en surface tandis qu'un noyau de miel à basse température subsiste au centre. Ce noyau, plus dense, tombe au fond du récipient et ne disparaît que lentement. Tandis que les courants de convexion contribuent à homogénéiser rapidement la température au sein du miel liquide la masse solide ne s'échauffe que lentement du fait de la très faible conductibilité calorifique du miel.

En second lien intervient la chaleur spécifique du miel. Herver l'a déterminée pour le miel liquide à I7 p. Ioo d'eau à $20^{\circ}$; elle est alors de 0,54 . Par contre on ignore la chaleur spécifique du miel solide ; il est possible qu'elle soit très sensiblement différente. On ignore également la valeur exacte de la chaleur de dissolution des cristaux de glucose dans la phase liquide. On sait simplement qu'elle intervient de façon notable dans le calcul de la quantité de chaleur nécessaire pour refondre un miel grâce à l'examen de la courbe de température obtenue en liquéfiant un petit échantillon de miel (fig. I) ; une nette inflexion apparait vers $40^{\circ}$ au moment où précisément les cristanx sont en cours de dissolution.

Comparativement au réchauffement d'une masse liquide (eau par exemple) le réchauffement d'une masse de miel pose des problènes beaucoup plus complexes. Ne pouvant les résoudre en totalité par le calcul faute de données théoriques suffisantes, nous en avons fait une étude empirique qui certes ne résout pas la question de façon définitive mais qui pourra apporter aux apiculteurs quelques éléments pratiques directement utilisables.

Matériel utilisé. -- Nous avons étudié dans ce travail la refonte du miel cristallisé dans une étuve électrique d'une puissance de $6 \mathrm{Kw}$ et d'un volume d'un mètre cube (dimensions intérieures : I $\times I \times I \mathrm{~m}$ ) soigneusement calorifugée et thermostatée $\left(^{1}\right)$. Nous disposions par ailleurs d'un appareillage électrique permettant d'enregistrer la température à $0,5^{\circ}$ près en 6 points différents de l'étuve par thermomètres à résistances de platine $\left({ }^{2}\right)$. Nous avions transformé plusieurs de ces thermomètres à résistance en cannes-sondes pouvant être introduites facilement dans la masse du miel à la profondeur désirée. La consommation de courant était appréciée par lecture d'un compteur du type courant donnant le demi-Kwh et ne desservant que l'étuve.

(1) Nous remercions ici la maison M. A. X. I.. I., 25 boulevard de Coubevoie a Nenilly-sur-Seine qui a mis à notre disposition l'étuve étudice.

(2) Nous remercions la maison Brion-Leroux pour le prêt gracieux de l'appareillage themométrique utilisé. 
Résultats obtenus. - Nous distinguerons parmi les essais réalisés :

Io I.es essais à vide destinés à évaluer les pertes calorifiques de l'étuve en fonctionnement.

$2^{\circ}$ Les essais de chauffage d'ean pure.

$3^{\circ}$ I es essais de refonte de quantités rariables de miel.

$I_{4}$ e tableau I résume l'ensemble de nos essais et contient toutes les données chiffrées ayant une valeur certaine. Des I7 essais réalisés nous $n^{\prime}$ 'en retiendrons que $I_{5}$ présentant un intérêt suffisant. I es essais $n^{0} 6$ et $n^{0} 7$ étant entachés d'erreurs par suite d'incidents divers n'ont pas été retenus.

Tous les essais, sauf le $n^{\circ}$ ith et le $11^{\circ}$ I 7 ont été conduits avec un réglage thermostatique identique assurant la coupure du courant vers $90-95^{\circ}$ et la remise en route vers $78-80^{\circ}$. Une sonde thermométrique placée dans l'étuve à proximité de la tige du thermostat rend compte pour tous les essais des alternances de chauffage et de refroidissement et permet de calculer le cycle de fonctionnement de l'étuve. Les essais 16 et I7 ont été effectués avec un réglage différent donnant l'écart de $60-62^{\circ}$ à $74-80^{\circ}$.

En règle générale, les 5 sondes thermométriques restant disponibles ont été introduites dans la masse du miel à réchauffer en des points différents : partie haute du fût de miel, partie centrale, partie basse. I orsque la masse était répartie en plusieurs bidons on prenait la température en divers récipients.

EssaIS DE L'ÉTCVE VIDE. - L'essai no il a été effectué avec le premier réglage du thermostat. En l'absence de toute charge la coupure du courant se produit seulement vers $100^{\circ}$. I a consommation est de $6 \mathrm{~K}$ wh (soit 5,2 Thermies) en 4 heures, soit I,5 Kwh ( $\mathrm{I}, 3 \mathrm{Th}$ ) par heure de chauffage Cette consommation représente uniquement les pertes calorifiques de l'étuve pour un écart de températures de $90^{\circ}-18^{\circ}=72^{\circ}$. On sait que les pertes sont proportionnelles à l'écart de température entre l'étuve et le milieu extérieur. Dans l'essai $n^{\circ}$ I 6 avec un réglage thermostatique donnant une température moyenne plus basse, les pertes horaires s'élèvent seulement à 0,75 Th par heure pour un écart de température de $70^{\circ}-20^{\circ}=50^{\circ}$. $\mathrm{L}$ a période de fonctionnement du thermostat est très différente selon le réglage : 6-26 minutes dans le premier cas contre 6-44 minutes dans le second. Ceci est parfaitement en accord avec la loi de proportionnalité des pertes à l'écart des températures.

EsSaIS DE CHAUHFAGE D'EAU PURE. - Dans l'essai $11^{0}$ I 5 nots avons chargé l'étuve avec 525 litres d'eau pure répartis en $\mathrm{r}_{5}$ bidons de 35 litres bien fermés pour limiter le plus possible la vaporisation. La figure 2 montre que l'élévation de température de l'eau se fait de façon à peu près linéaire. La diffusion de la chaleur dans le milieu liquide est suffisamment rapide grâce aux courants de convexion pour que le thermostat 


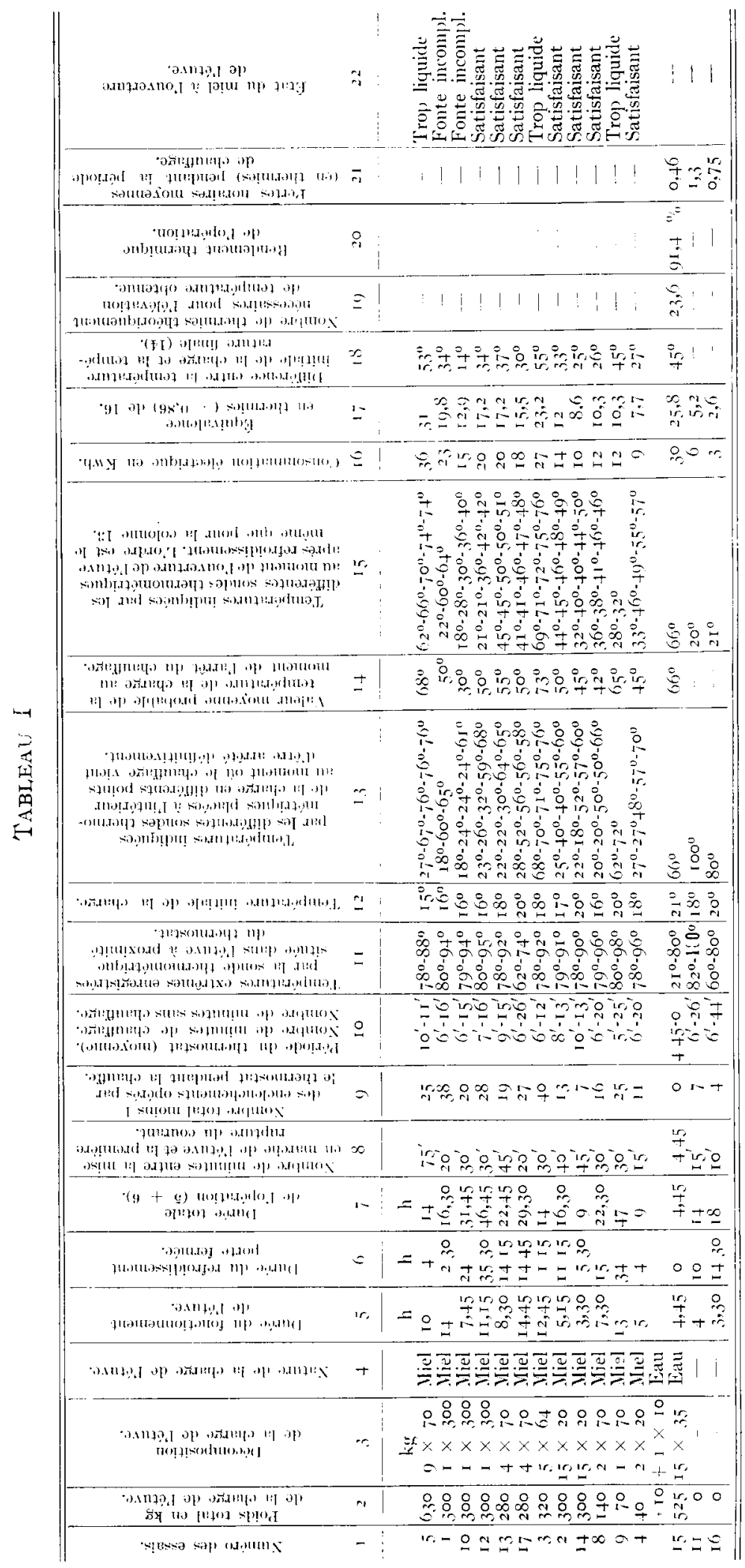


ne coupe jamais le courant. I e bilan thermique, facile à établir puisqu'on connaît la chaleur spécifique de l'eau s'établit comme suit :

Chaleur utilisée : $30 \mathrm{Kwh} \times 0,86=25,8 \mathrm{Th}$;

Chaleur nécessaire : $0,5^{2} 5 \times \mathrm{I} \times\left(66^{\circ}-2 \mathrm{I}^{\circ}\right)=2,3,6^{\prime} \mathrm{Th}^{\mathrm{T}}$;

Rendement : $\frac{23,6 \times \text { IOO }}{25,8}=91,41$ ). 100

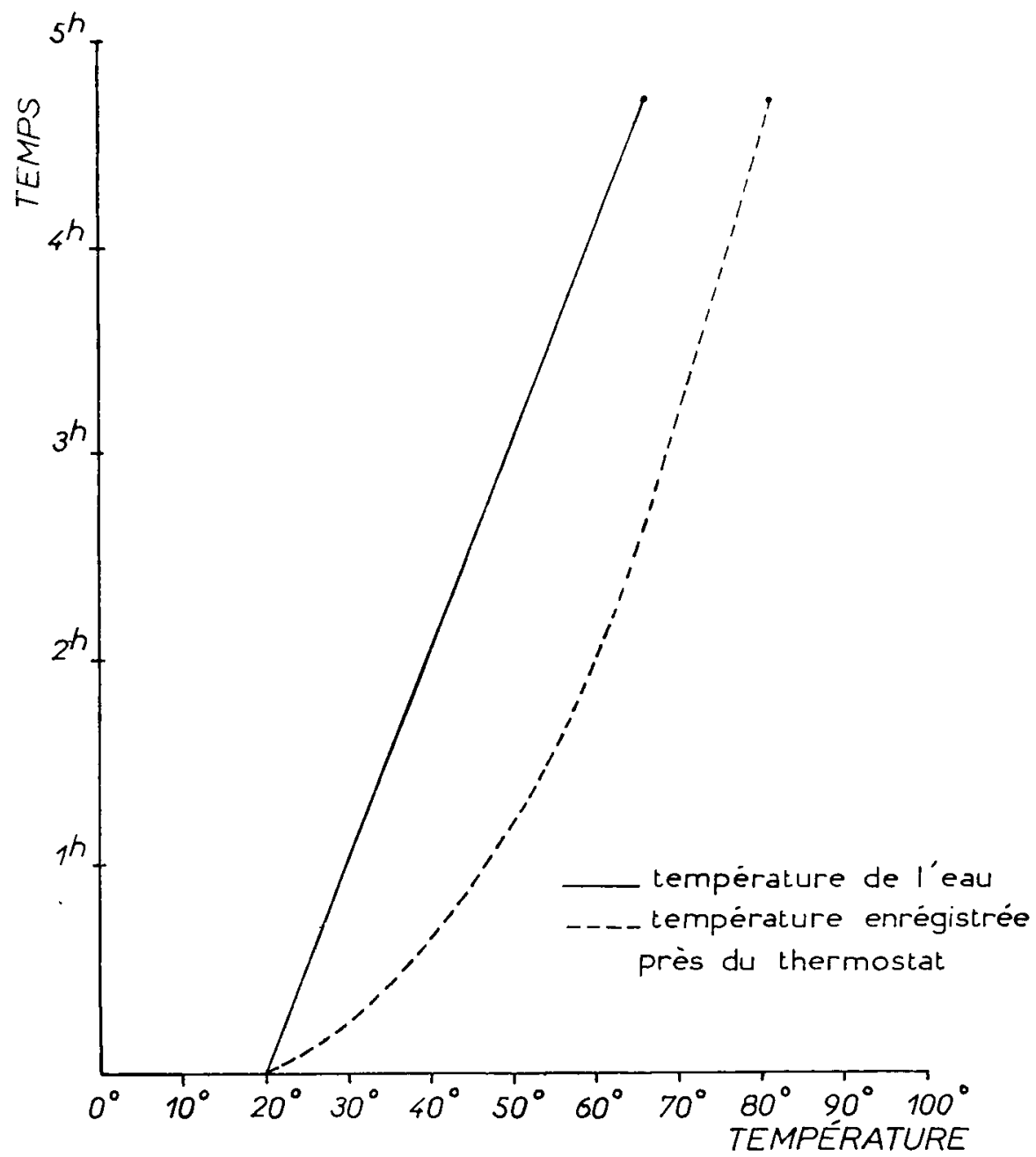

Fic. 2. Explication dans le texte.

On ne tient pas compte de la chaleur nécessaire pour échauffer les récipients métalliques et qui peut facilement être négligée parce que peu importante. I,es pertes calorifiques horaires de l'éture sont de :

$$
\frac{25,8-23,6}{4,75}=0,46 \text { Th par heure en moyenne. }
$$


I ans ces conditions de fonctionnement le rendement de l'opération est excellent. On notera que l'ensemble éture-charge reste toujours dans des limites de températures assez basses, ce qui explique le taux éleré din rendenent.

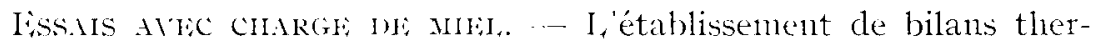
miques précis est impossible lorsqu'il s'agit de miel solide pour les raisons que nous avons indiquées plus hant. Par ailleurs il est impossible de connaitre la température finale du miel car au moment de l'arrêt du chauffage on a toujours en présence dans le menne récipient des nuasse: de miel de poids inconnu ì des températures très différentes. Il n'est possible que l'évaluer grossièrement la température moyenue en se basant sur les indications fournies par les differentes sondes thermonttriques. Par contre il est possible de noter l'état final clu miel à l'ouverture de l'éture : refonte totale on partielle, chanfage excessif ou non. On peut aussi connaître la consommation de conrant électrique et finalement comparer entre elles des opérations comportant nue on denx variables.

I)'une façon très générale, l'examen des courbes obtenues par enregistrement de la température en divers points de la masse de miel montre une très faible variation de température dans le niel solide qui ne s'échauffe. qu arec une grande lenteur. Dès que la sonde est atteinte par un flot de miel liquide la tenuérature monte très rapidennent d'un seul coup puis se stabilise. linfin, l'échauffement se prolongeant la température du miel liquide augmente d'une façon progressive juscqu'à l'arrêt du chauffage (fig. 3). I orsqu'on refond du miel contenu dans un récipient de grande capacité on observe couramment, au moment où l'on interrompt $k^{\circ}$ chauffage des écarts de température de l'ordre de 30 à $40^{\circ}$ entre les points les plus chauds (roisinage les parois) et les points froids représentés par du miel non encore fondu. Au cours du refroidissement, l'étuve restant fermée, il y a égalisation des températures par échanges thermiques et. en général le dernier noyau solide parvient à l'état liquide à ce moment. I.'enregistrement permet de suivre tous ces phénomènes avec précision.

I. examen du tableau I nontre que nous avons essayé de comparer la rentabilité d'opérations de refonte arec comme variables :

Io I a charge de l'éture.

$2^{\circ}$ I, 'état de division de cette charge.

$3^{\circ}$ I e rêglage thermostatique.

Fxaminons donc successivenent l'importance des ces variables.

$\mathrm{I}^{\circ}$ Influence de la charge. - I a comparaison des essais $n^{\circ} 5$, I $2, \mathrm{I}_{3}$. S' et 9 met en évidence la constatation suivante: plus la charge de l'éture est forte plus l'opération de refonte est rentable. Comparons les résultats.

Essai $11^{\circ} 5: 630 \mathrm{~kg}$ sont refondus et portés à nue température trop, élevée (680 en moyenne) pour 3 I 'Th. 


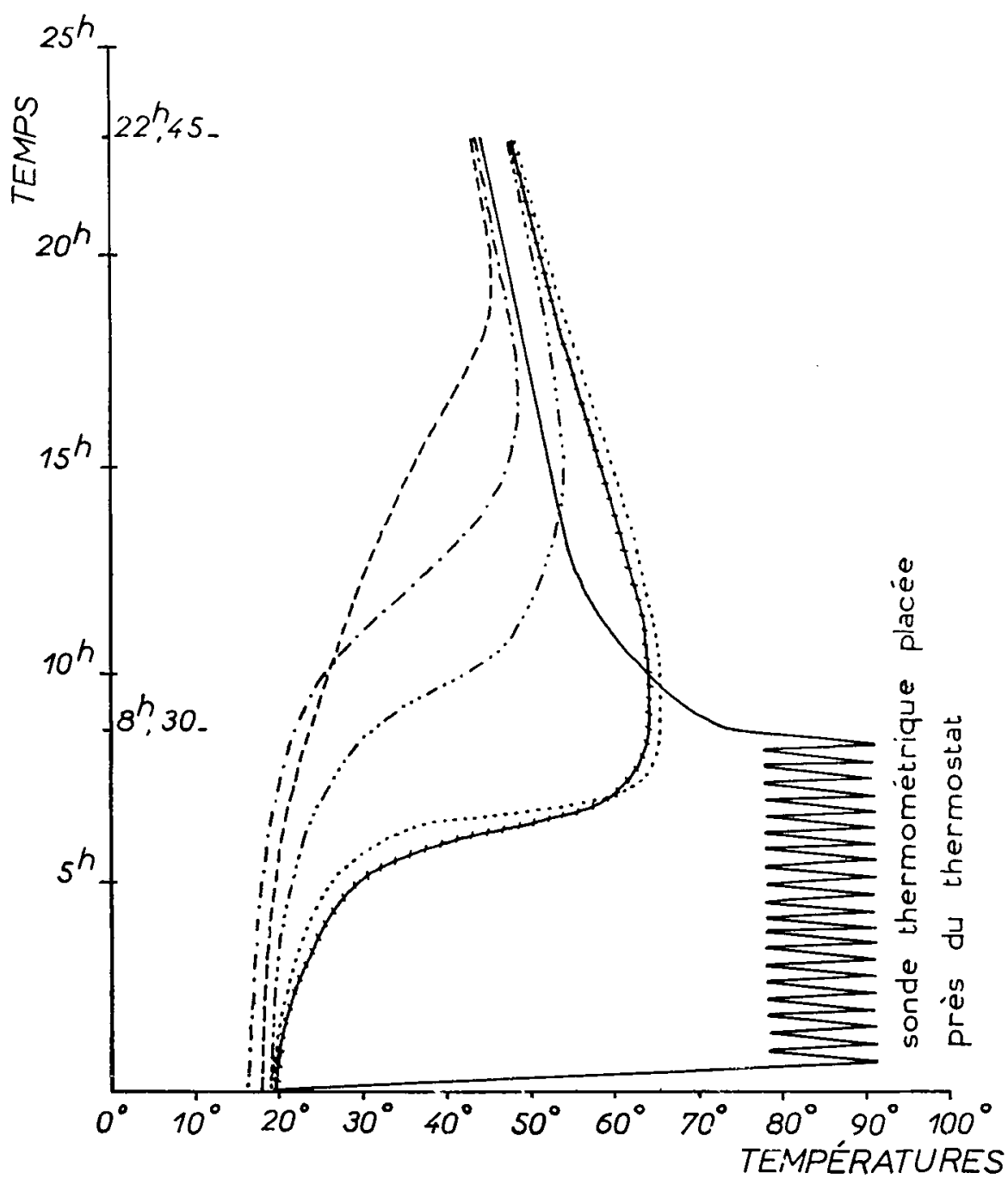

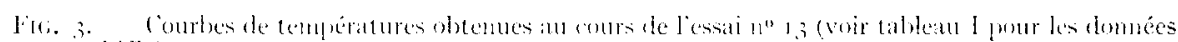
chiffries).

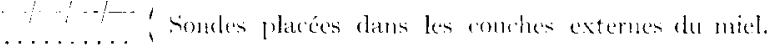
Sonde movenne.

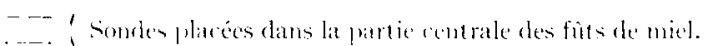

Issai $n^{\circ}$ I $2: 300 \mathrm{~kg}$ sont portés à $50^{\circ}$ environ pour I7,2 'Th.

Fssai $n^{\circ} \mathrm{I} 3: 280 \mathrm{~kg}$ sont portés à $55^{\circ}$ environ pour $\mathrm{I} 7,2$ 'Th.

Fissai $11^{\circ} 8$ : $140 \mathrm{~kg}$ sont portés à $42^{\circ}$ entiron pour Io, 3 'Th.

Isssai $1^{\circ} 9: 70 \mathrm{~kg}$ sont portés à $65^{\circ}$ environ pour 10,3 'Th.

Compte tenu des irrégularités inévitables dans la température finale du micl et si l'on compare senlementut les essais extrênes 5 et 9 , on constate 
que pour une élévation de température de $50^{\circ}$ environ $\left(45^{\circ}\right.$ à $\left.53^{\circ}\right)$ il a fallu ro Th pour $70 \mathrm{~kg}$ contre 3I Th pour $630 \mathrm{~kg}$, donc seulement 3 fois plus d'énergie pour 9 fois plus de poids. Les pertes calorifiques, qui sont relativement fixes, sont donc considérables par rapport à l'énergie effectivement utilisée lorsque la charge est faible.

$2^{0}$ Influence de la division de la charge. - On comparera utilement à ce sujet les essais $n^{\circ}{ }^{2} 2, I_{3}, 2$ et 14 qui portent tous sur une masse de miel de l'ordre de $300 \mathrm{~kg}(280$ à $300 \mathrm{~kg})$.

Essai no I2: $300 \mathrm{~kg}$ en I fût, bien fondu $\left(50^{\circ}\right)$ pour I7,2 Th.

Esssai $11^{\circ} I_{3}: 280 \mathrm{~kg}$ en 4 fûts, bien fondu $\left(55^{\circ}\right)$ pour $17,2^{\text {Th }}$.

Essai $n^{0} 2: 300 \mathrm{~kg}$ en 15 fûts, bien fondu $\left(5^{\circ}\right)$ pour $12 \mathrm{Th}$.

Essai $n^{0} 4: 300 \mathrm{~kg}$ en $\mathrm{I} 5$ fûts, bien fondu $\left(45^{\circ}\right)$ pour $8,6 \mathrm{Th}$.

On constatera que plus la masse est divisée moins la quantité de chaleur nécessaire est grande. Ceci s'explique parfaitement par le fait que la conductibilité calorifique du miel solide est très faible. I a fonte d'une masse unique de $300 \mathrm{~kg}$ est plus lente que celle d'une masse très divisée; les pertes calorifiques étant proportionnelles au temps, elles sont plus importantes dans le premier cas.

I e gain de calories dans le cas d'une masse très divisée peut atteindre près de $5^{\circ} \mathrm{p}$. Ioo. I a comparaison des essais $\mathrm{n}^{\circ} 2$ et $\mathrm{I}_{4}$ montre bien que ce sont les calories les plus chaudes qui sont les plus coûteuses; une différence de température finale de l'ordre de $5^{\circ}$ se traduit par un excédent de consommation important.

$3^{0}$ Influence de la température de l'étuve. - Si l'on compare les essais I3 et I7 on constate que la refonte à $60-80^{\circ}$ est un peu plus économique que la refonte à $80-90^{\circ}$ mais la température finale n'étant pas exactement la même il est difficile de tirer une conclusion précise. Le temps de chauffe est beaucoup plus longdans le premier cas que dans le second ; l'économie faite en utilisant des calories à température plus basse semble bien compenser sensiblement l'excédent de consommation dû̀ des pertes calorifiques plus prolongées. On remarquera encore que les températures finales sont mieux équilibrées dans l'essai $n^{\circ}$ I 7 et que les surchauffes locales sont moins importantes. Notons encore que malgré les surchauffes locales les miels soumis aux essais ont tous repris l'état solide dans de bonnes conditions, ce qui prouve qu'ils étaient encore riches en germes de cristallisation.

Prix de revient de la refonte du miel. - Selon l'importance et la division du chargement de l'étuve, selon le réglage thermostatique, selon le tarif dont bénéficie l'utilisateur auprès de l'F. D. li., le prix de revient. de la refonte d'un kilo de miel (passage de $18^{\circ}$ à $45^{\circ}$ ) peut varier de :

$$
\left(\frac{\mathrm{Kwh} \times \mathrm{Fr}}{\mathrm{Kg}}\right) \frac{31,5 \times 6}{630}=0,30 \mathrm{Fr} \text { à } \frac{\text { Io } \times 10}{70}=1,40 \mathrm{Fr}
$$


avec tous les intermédiaires. D’une façon générale et en considérant une organisation rationnelle du travail on peut estimer que le prix au $\mathrm{kg}$ de la refonte par chauffage électrique ne doit pas revenir à plus de 0,75 fr à I fr au grand maximum. I, 'utilisation de courant de nuit à bas prix doit permettre en beaucoup de régions un prix de revient très bas.

Bien entendu il conviendrait de tenir compte de l'amortissement de l'étuve. Calculé sur Io ans cet amortissement ressort à 30 ooo fr par an pour l'étuve que nous avons utilisée. I, 'achat d'un tel appareil ne semble rentable que si l'exploitation doit réchauffer chaque année Io à 20 tonnes de miel au moins. I1 faut fondre 30 à 40 tonnes de miel par an pour que l'amortissement soit égal ou inférieur au prix du courant consommé. Beaucoup d'apiculteurs envisageront donc une installation plus économique au départ et nous donnerons dans nos conclusions les directives générales permettant de réaliser un système qui convienne à chaque exploitation.

Conclusions. - La refonte du miel par un dispositif de chauffage électrique se révèle comme parfaitement valable à tous égards dans la mesure où l'on observe les règles générales suivantes :

I. I,es pertes calorifiques représentant l'obstacle essentiel à un travail rentable, il convient de les limiter le plus possible. Pour ce faire on remarquera :

a) que la déperdition par les parois d'une étuve ou d'une chambre chauffante quelconque est fonction de la surface de ces parois, de leur coefficient de conductibilité calorifique, et de la différence de température entre le milieu interne et le milieu externe;

b) que la surface crôit avec le carré des dimensions et le volume avec leur cube. Les pertes calorifiques dont donc relativement plus faibles dans une grande étuve que dans une petite à calorifugeage égal ;

c) qu'il convient de proportionner la charge de l'étuve à son volume utile; plus la charge est voisine du maximum, plus le rendement calorifique est élevé ;

d) qu'il est avantageux de diviser le plus possible la charge. Le fût de $70 \mathrm{~kg}$, d'une manipulation aisée, présente pour la refonte un gros avantage sur le fût de $300 \mathrm{~kg}$ exigeant une main-d'cuvre accrue pour sa manipulation et d'un réchauffement plus onéreux et plus délicat ;

e) qu'on doit rechercher un règlage thermostatique (') judicieusement calculé et que nous estimons se situer vers 60 à $70^{\circ}$ au maximum de préférence à une température trop élevée amenant l'apparition de points chauds dans le miel, ou trop basse $\left(40^{\circ}\right)$ qui a l'inconvénient de prolonger par trop 1'opération de refonte, ce qui est également nuisible au miel.

( ${ }^{1}$ Il convient de bien noter que le réglage thermostatique de l'ćtuve à $60-70^{\circ}$ n'implique pas que tout le miel réchauffé sera porté à $60-70^{\circ}$. On doit toujours s'efforcer d'obtenir une température finale moyenne du miel de l'ordre de $45^{\circ}$. 
2. Des réalisations écononiques peuvent être envisagées par les apiculteurs en fonction de leurs besoins. Il est toujours possible de réaliser au moyen de matériaux peu coûteux une enceinte soigneusement calorifugée; l'achat des éléments chauffants et des appareils de réglage de la température ne représente plus qu’une dépense assez modique supportable par n'importe quelle exploitation moyenne.

\section{REFGRFNCES BIBLIOGRAPHIQUES}

(I) Hus.vey ('T. ('.). . - Study on some physical properties of honey. Fond Reseurch, 19 (3), 282-20)2, 1954. 